Questions vives

\section{Questions Vives}

Recherches en éducation

Vol. $5 n^{\circ} 11 \mid 2009$

Le développement professionnel : quels indicateurs?

\title{
La socialisation au travail comme indicateur de développement professionnel : analyse des approches basées sur la mesure
}

Stéphane Martineau, Liliane Portelance et Annie Presseau

\section{(2) OpenEdition \\ 1 Journals}

Édition électronique

URL : http://journals.openedition.org/questionsvives/614

DOI : $10.4000 /$ questionsvives.614

ISBN : 978-2-8218-1082-2

ISSN : $1775-433 \mathrm{X}$

Éditeur

Université Aix-Marseille (AMU)

Édition imprimée

Date de publication : 1 janvier 2009

Pagination : 243-258

ISBN : 978-2-912643-35-3

ISSN : $1635-4079$

\section{Référence électronique}

Stéphane Martineau, Liliane Portelance et Annie Presseau, «La socialisation au travail comme indicateur de développement professionnel : analyse des approches basées sur la mesure ", Questions Vives [En ligne], Vol. $5 \mathrm{n}^{\circ} 11$ | 2009, mis en ligne le 01 juin 2011, consulté le 08 janvier 2020. URL : http:// journals.openedition.org/questionsvives/614; DOI : 10.4000/questionsvives.614

\section{(c) (i) (9)}

Questions Vives est mis à disposition selon les termes de la licence Creative Commons Attribution Pas d'Utilisation Commerciale - Pas de Modification 4.0 International. 


\title{
La socialisation au travail comme indicateur de développement professionnel : analyse des approches basées sur la mesure
}

\author{
Stéphane Martineau, Liliane Portelance, Annie Presseau ${ }^{1}$
}

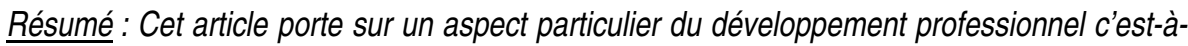
dire la socialisation professionnelle (ou organisationnelle). Plus spécifiquement, ce sont certains des aspects méthodologiques des recherches qui sont ici étudiés. Ainsi, les auteurs conduisent une réflexion critique sur les différents outils de mesure les plus utilisés dans les recherches sur la socialisation professionnelle. Les principales insuffisances des recherches sont identifiées et des pistes de perfectionnement des outils sont esquissées. Ils s'interrogent par ailleurs sur la pertinence des approches basées sur la mesure du processus de socialisation professionnelle.

Mots-clés : socialisation professionnelle, méthodologie, développement professionnel

Abstract: This article focuses on a particular aspect of professional development which is professional or organizational socialization. More specifically, some methodological aspects of research on professional socialization are studied here. Thus, the authors conduct a critical analysis on the various measurement tools used in the research on professional socialization. The main shortcomings of the research are identified and possible development tools are outlined. In addition, they also question the relevance of the approaches based on the measure of the process of professional socialization.

Keywords: professional socialisation, methodology, professional development

\footnotetext{
${ }^{1}$ Professeurs en Sciences de l'Éducation - Université du Québec à Trois-Rivières (UQTR) - Centre de recherche interuniversitaire sur la formation et la profession enseignante (CRIFPE) - Laboratoire d'analyse du développement et de l'insertion professionnels en enseignement (LADIPE)
} 


\section{La socialisation au travail comme indicateur de développement professionnel : analyse des approches basées sur la mesure}

\section{Introduction}

Notre texte se veut pour l'essentiel une analyse de quelques aspects méthodologiques et épistémologiques des recherches sur la socialisation professionnelle (ou organisationnelle), recherches liées au champ du développement professionnel. Plus précisément, nous y conduisons une réflexion critique sur les différents outils de mesure les plus utilisés en recherche dans ce domaine. D'abord, nous esquissons une problématique de la recherche en socialisation professionnelle et nous apportons quelques précisions conceptuelles nécessaires en situant la question de la socialisation par rapport au développement professionnel. Ensuite, nous spécifions nos critères d'analyse de la littérature spécialisée. Suit alors une analyse critique des aspects méthodologiques des recherches sur la socialisation professionnelle. Les principales insuffisances des recherches sont identifiées notamment au regard des indicateurs - et des pistes de perfectionnement des outils sont esquissées. Enfin, nous menons une brève discussion sur la pertinence épistémologique des approches de la mesure dans l'analyse de la socialisation professionnelle.

\section{Mise en contexte}

Dans le domaine de la socialisation professionnelle (parfois nommée aussi socialisation organisationnelle) les recherches ont connu un développement que l'on peut qualifier de paradoxal. Ainsi, depuis trois décennies environ les chercheurs ont clairement démontré que la socialisation professionnelle est un enjeu central pour la compétence des acteurs en milieu de travail. Plus spécifiquement, on sait qu'en enseignement, les milieux scolaires qui mettent sur pied des dispositifs d'insertion professionnelle favorisent une meilleure entrée dans la carrière enseignante et réduisent les risques de décrochage de la profession (Martineau, Presseau \& Portelance, 2009; Martineau \& Vallerand, 2007). Toutefois, et c'est là le paradoxe que nous signalions au début de ce texte, la grande richesse dans l'analyse des indicateurs et des implications de la socialisation professionnelle s'accompagne aussi de lacunes importantes tant sur le plan de la définition du concept que sur celui de sa mesure dans la tradition des recherches reposant sur la mesure.

La définition de la socialisation professionnelle ne fait pas consensus. Pour certains, elle permet de maîtriser un rôle en milieu de travail (Van Maanen \& Schein, 1979). Pour d'autres la socialisation professionnelle permet une compréhension de la culture d'une organisation (Louis, 1980). Enfin, pour d'autres encore, elle renvoie au phénomène d'appartenance à une organisation (Feldman, 1976). Si les définitions abondent (et ici nous ne pouvons citer tous les auteurs consultés), on en connaît beaucoup moins sur la nature du processus en tant que tel ; bien qu'à cet égard les travaux de Dubar (1996) et de Dubar et Tripier (2005) ont indiqué des pistes prometteuses. En fait, la socialisation professionnelle apparaît comme étant un processus défini essentiellement par ses résultats plutôt que par son fonctionnement (nous y reviendrons). Par ailleurs, les chercheurs sont loin de s'entendre au sujet de la nature de ces résultats, de sorte qu'un certain flou demeure. 
Le développement professionnel : quels indicateurs?

\section{a) Des défis pour la recherche}

Revenons d'abord rapidement sur les sources des théories de la socialisation. Très tôt deux logiques se sont fait concurrence. D'un côté nous avons les thèses déterministes où la socialisation est intimement liée à la perpétuation des sociétés (Bolliet \& Schmitt, 2002). Ici, le regard se porte d'abord sur la société et la socialisation est, pour l'essentiel, un processus de transmission de la culture (Durkheim, 1967; Linton, 1986; Rocher, 1992). D'un autre côté, on retrouve les thèses essentiellement individualistes (Weber, 1971). Pour elles, la socialisation est d'abord un processus de formation de la personne (Piaget, 1965). Distinguons les succinctement (le lecteur nous pardonnera ici une schématisation trop rapide).

Les approches holistes ou déterministes reposent en quelque sorte, plus ou moins explicitement, sur les idées suivantes:

-l'homme apprend de manière essentiellement passive par intériorisation;

-l'homme est façonné par la société ;

-il y a primauté de la société sur l'individu ;

- la société impose des valeurs, des normes, des rôles qui exercent une contrainte sur les

-individus ;

-l'action de l'individu est conditionnée (agents de socialisation : famille, école).

En conséquence, la recherche met surtout en évidence le conformisme aux rôles, aux valeurs, aux normes, aux attitudes.

Quant à elles, les approches plutôt individualistes (inspirées plus ou moins de la sociologie compréhensive de tradition wébérienne, de l'interactionnisme et de la psychologie sociale) mettent de l'avant les éléments suivants :

-l'homme apprend de manière essentiellement active par appropriation personnelle;

-la société est façonnée par l'homme ;

-il y a primauté de l'individu sur la société ;

- les normes, les valeurs et les rôles ne sont que des possibilités offertes à l'individu qui conserve une marge de liberté dans l'exercice de ces rôles.

Ici, l'individu est un acteur social. II « agit sur » autant « qu'il est agi par » le monde social dans lequel il évolue.

Les tentatives pour dépasser l'enfermement dans l'une ou l'autre des deux logiques ont été nombreuses; on pense notamment, pour le processus général de socialisation en société, aux travaux pionniers de Mead parus dans les années 1930 (1963). La théorie de la structuration de Giddens en est un exemple relativement récent (2005, première édition en français 1987). Toutefois, à l'heure actuelle, il n'existe pas encore de théorie générale de la socialisation qui puisse réconcilier les diverses positions (Lacaze, 2002). En cela, il s'agit, convenons-en, d'une situation normale pour les sciences humaines et sociales où la diversité d'approches, de théories et de paradigmes est de mise et notre texte n'a aucune prétention de jouer un quelconque rôle rassembleur. Notre propos est considérablement plus modeste.

Ce même clivage entre visions plutôt holistiques ou déterministes et visions principalement individualistes se vérifie dans les recherches plus spécifiques sur la socialisation professionnelle. Par exemple, si certains voient la socialisation professionnelle comme un processus cognitif d'attribution de sens (Louis, 1980), d'autres l'identifient davantage à une stratégie organisationnelle pour « enculturer » le travailleur (Van Maanen \& 
Stéphane Martineau, Liliane Portelance, Annie Presseau

La socialisation au travail : analyse des approches basées sur la mesure

Schein ; 1979). Pour leur part, Sainsaulieu $(1977,1984)$ et Dubar (1996) mettent l'accent sur les interactions au travail lesquelles seraient centrales dans la structuration de l'identité professionnelle. Ces deux sociologues, influencés par le courant interactionniste, insistent sur le fait que la culture organisationnelle que doit connaître le nouveau travailleur se modifie sans cesse. Par ailleurs, leurs travaux laissent bien voir qu'au sein d'une organisation, on retrouve différentes identités professionnelles qui renvoient à autant de catégories de " travailleurs » différents. Par exemple, dans une école, on n'a qu'à penser aux enseignants, aux professionnels non enseignants (psychologues scolaires, etc.), aux membres de la direction (Sainsaulieu, 1984 ; D'lribarne, 1989, 1986).

Cette diversité d'approches n'est pas sans présenter un défi. L'absence de consensus au sujet de la socialisation rend en effet son opérationnalisation difficile. Dès 1986, Fisher fait remarquer qu'au moment où il écrit, non seulement les recherches sur la socialisation professionnelle ont donné lieu à un faible nombre d'études empiriques mais, surtout, que les mesures - lorsqu'elles existent - portent sur des indicateurs indirects. En fait, les recherches mesurent les conséquences attendues de la socialisation professionnelle. Ces premières recherches utilisent donc les indicateurs suivants : satisfaction, engagement, performance. Des recherches ultérieures ont essayé de dépasser le relatif simplisme des premières études en se centrant sur l'identification de conséquences plus directes de la socialisation professionnelle. Elles ont donc tenté d'identifier les éléments qui devraient être maîtrisés à l'issue du processus de socialisation au travail. Ces recherches ont eu le mérite d'étudier le contenu même de la socialisation et d'établir des indicateurs spécifiques au concept. D'autres recherches encore, en privilégiant une analyse du processus même de la socialisation professionnelle, ont défini différents stades que le travailleur traverse durant sa socialisation professionnelle. Nous y reviendrons plus loin.

\section{b. Quelques définitions du concept}

Bien des chercheurs se sont penchés sur cette question de la socialisation professionnelle en particulier dans divers corps d'emploi allant des enseignants aux entraîneurs sportifs en passant par les gardiens de prison. Ces chercheurs ont alors proposé différentes définitions de la socialisation professionnelle. À titre indicatif voyons-en brièvement quelques unes.

Selon Dixon $(2005,14)$ : "Professionnal socialization is a continous process of adaptation to and personalization of one's environment ". Quant à Helm $(2004,76)$, il soutient : " The process through which individuals gain the knowledge, skills, and value necessary for entry into a professional career an advanced level of specialized knowledge and skills ". Clark $(1997,442)$ va un peu dans le meme sens: " acquisition of the knowledge, skills, value, roles, and attitudes associated with the practice of a particular profession ". Pour ce qui est de Klossner $(2004,12)$, il propose ce qui suit: « process by which individuals learn the roles and responsibilities of their profession and become emerging members of the professional culture ". Pour leur part, Dunn, Linda and Seff $(1994,375)$ diront que la socialisation professionnelle est un processus : " by which individuals acquire the attitudes, beliefs, values and skills needed to participate effectively in organized social life ". Spécifiquement, en ce qui a trait aux enseignants, Lacey $(1994,6122)$ affirme que la socialisation professionnelle: "refers to the process of change by which individuals become members of the teaching 
Le développement professionnel : quels indicateurs?

profession and then take on progressively more mature roles, usually of higher status, within the profession ".

Au delà des différences de définitions plusieurs caractéristiques communes ressortent de ce bref tour d'horizon. D'abord, la socialisation professionnelle est un processus continu (Hébrard, 2004) dont la fin, ultimement, n'est envisageable qu'au moment où l'employé quitte l'organisation (par exemple, à la retraite). Ce processus est complexe et comprend des aspects tant cognitifs, affectifs qu'interactifs (Dubar, 2000 ; Gundry, 1993). II prend forme dans l'interaction entre l'acteur et son environnement physique et social de travail (Adler \& Adler, 2005 ; Shamatov, 2005). II se traduit par l'acquisition d'une sorte de culture de l'institution (ou de l'organisation) vérifiable notamment à travers le rapport à certaines valeurs, la possession de certaines connaissances et la mobilisation de certaines compétences (Allen \& Meyer, 1990; Ashford \& Saks, 1996; Dixon, 2005). C'est dire que la socialisation professionnelle se vérifie notamment dans l'attitude et la pratique du travailleur (Høivik, 2005 ; Keith \& Moore, 1995). Enfin, la socialisation professionnelle comporte des incidences certaines sur l'identité professionnelle de l'acteur (Klossner, 2004 ; Martineau, 2008). En cela, elle est un processus de changement identitaire où le sujet se définit par rapport à son groupe professionnel (Langlois, 2000 ; Osiek-parisod, 1995).

\section{Le développement professionnel}

Après ce bref rappel des questions entourant la problématique de la socialisation professionnelle, jetons un œil sur le développement professionnel. II s'agit d'un concept qui a émergé d'abord dans les recherches de type managérial et dont la popularité a été croissante, ces dernières décennies, au fur et à mesure que le marché du travail s'engageait dans une restructuration majeure axée notamment sur la polyvalence de la main-d'œuvre et sa responsabilisation face à la qualité du travail fourni (Bryant, 2007). C'est dans cette même optique, par exemple, que le concept de compétence a pris le pas sur celui de qualification. II en existe plusieurs définitions. Killion $(2002,11)$ soutient ainsi que le développement professionnel est «...the planned, coherent actions and support systems designed and implemented to develop knowledge, skills, attitudes, aspiration, and behaviors to improve student achievement ». Pour sa part, Guskey $(2000,16)$ précise que le développement professionnel inclut : « ...those processes and activities designed to enhance the professional knowledge, skills, and attitudes of educators so that they might, in turn, improve the learning of students ». Ou encore, pour Nault $(2005,30)$ : " Le développement professionnel est la somme des apprentissages effectués de façon formelle ou informelle au cours de la carrière, de ses débuts jusqu'à la retraite ». Nous pourrions citer ici encore bien des auteurs. Disons pour le moment que s'il y a un relatif consensus pour faire du développement professionnel un processus dans lequel le travailleur s'engage afin d'en arriver à un niveau de maîtrise et de compréhension supérieur de sa pratique (Uwamariya \& Mukamurera, 2005), on constate également des divergences quant à la manière de définir ce processus. Ces divergences vont porter sur l'ampleur du processus : transformation des seuls savoirs et compétences ou modification également des attitudes voire des aspirations professionnelles. Elles vont porter aussi sur la manière dont le développement est assuré : d'aucuns soutiennent que le développement professionnel ne saurait se faire de manière informelle pendant que d'autres vont jusqu'à inclure l'apprentissage informel comme modalité de développement (Nault, 2005 ; Day, 1999). Enfin, certains vont insister sur la dimension collaborative du processus 
Stéphane Martineau, Liliane Portelance, Annie Presseau

La socialisation au travail : analyse des approches basées sur la mesure

(Speck \& Knipe, 2001) pendant que d'autres mettront l'accent sur l'engagement personnel (Guillemette, 2006).

\section{La socialisation professionnelle dans le développement professionnel}

D'entrée de jeu, disons d'emblée qu'il va de soi que nous ne pourrons qu'esquisser ici les liens entre ces deux processus. D'abord, soulignons que, selon nous, la socialisation professionnelle est un processus qui semble plus global que celui de développement professionnel. En effet, tout acteur au travail s'inscrit dans un processus de socialisation où il est à la fois réceptacle des influences et agent de socialisation à son tour. On le sait, l'homme ne peut entrer en relation avec autrui, agir dans un contexte donné ou inscrire son activité professionnelle dans un cadre particulier, sans que de «la socialisation ne s'en suive ". En ce sens, la socialisation professionnelle est un processus qui englobe tout ce qui permet de maîtriser un rôle en milieu de travail, assure une certaine compréhension de la culture d'une organisation ou encore, définit un certain rapport identitaire à une organisation (Martineau \& Presseau, 2007). Quant au développement professionnel, il renvoie essentiellement, comme on la vu plus haut, à un apprentissage formel ou informel visant la plus grande maîtrise de l'agir professionnel. On peut dire alors que son horizon est finalisé : accroître l'efficacité et l'efficience de l'acteur dans son milieu de travail. Ainsi, si le concept de socialisation professionnelle peut, à la limite, se conjuguer tout autant dans le sens de l'intégration que de la distance (voire l'hostilité) aux objectifs de l'organisation - car on peut imaginer une socialisation délinquante, des comportements d'évitement " appris ", un " monde parallèle " des travailleurs à l'abris des supérieurs - on imagine mal un développement professionnel qui conduirait le travailleur à moins de bien-être au travail, à moins d'efficacité, à moins de professionnalisme.

On l'aura alors compris, les recherches, à majorité anglo-saxonnes, qui portent sur la socialisation professionnelle envisagent en général ce processus sous le seul angle de ce que nous pourrions considérer comme le développement professionnel. En effet, la grande majorité de ces recherches conçoivent la socialisation professionnelle dans une optique qu'il est possible de qualifier de normative au sens que la socialisation y est présentée sous l'angle de la meilleure intégration du travailleur à l'organisation. Cette orientation générale est directement liée aux origines managériales de ces recherches.

Avant de conclure cette section, il s'avère nécessaire de mieux spécifier en quoi la socialisation peut être un indicateur du développement professionnel. Au sens fort du terme, la socialisation est un processus de transformation du sujet qui s'approprie une culture donnée (en contexte de travail, la culture organisationnelle). La conséquence la plus saillante de la socialisation est de rendre relativement stables certaines dispositions (manière de sentir, de penser, d'agir). Or, la socialisation, bien qu'elle soit un processus individuel (chacun en fait une expérience personnelle et originale) conduit les sujets d'une communauté (de travail ou non) à un plus ou moins grand partage de valeurs, de règles, de normes, de représentations. En tant d'instrument de régulation sociale, la socialisation permet en outre l'économie de la surveillance et des sanctions externes au sens où les acteurs conforment leurs comportements aux attentes du groupe.

Ajoutons que dans le cadre d'une organisation de travail, lorsqu'elle s'oriente vers une certaine conformité à la culture organisationnelle, la socialisation professionnelle peut être vue comme un facteur de développement professionnel dans la mesure où le sujet acquiert 
Le développement professionnel : quels indicateurs?

une capacité à "lire les situations ", à se mouvoir adéquatement dans l'organisation, à adopter les bons comportements aux bons moments (en cela, il devient donc en quelque sorte plus efficace et plus efficient). On comprendra alors que toute organisation de travail gagne à abriter en son sein des acteurs " bien socialisés ». Mentionnons toutefois que le lien entre socialisation professionnelle et développement professionnel est loin d'être simple et automatique. En effet, on peut imaginer qu'une trop grande conformité aux normes et aux règles de l'organisation puisse conduire à des comportements ritualisés peu efficaces et qui laissent peu de place à l'innovation. Au fond, la socialisation professionnelle participera du développement professionnel dans la mesure où elle n'enfermera pas le sujet dans des rôles et des statuts stéréotypés mais lui permettra de développer un rapport réflexif à son travail. Malheureusement, nous ne pouvons ici développer plus avant ces quelques considérations et nous sommes conscients d'en rester à un niveau de généralité très élevé.

\section{Critères d'analyse d'un instrument de mesure en sciences humaines et sociales}

Notre questionnement de fond, rappelons-le, porte ici sur les indicateurs de la socialisation professionnelle. Plus spécifiquement, à partir d'une analyse de la littérature francophone et anglo-saxonne, analyse qui, il va sans dire, ne saurait être exhaustive, nous souhaitons réfléchir sur les instruments de mesure de la socialisation professionnelle. En effet, parler d'indicateurs renvoie immédiatement à leur opérationnalisation. En somme, nous nous posons la question suivante: les instruments mesurent-ils bien le concept de socialisation professionnelle?

On le sait, le social ne saurait se mesurer comme les objets physiques (Freitag, 2002). Les phénomènes sociaux sont complexes et les acteurs qui y participent en ont déjà une interprétation (Dumont, 1968). Giddens (2005) dirait qu'ils possèdent une conscience réflexive sur le monde qu'ils habitent. Par ailleurs, souvent les mesures ne peuvent être répétées dans le même contexte puisque les recherches ne se mènent pas en laboratoire. En fait, le problème central qui se pose aux chercheurs est d'identifier des indicateurs observables qui restituent le plus fidèlement possible les caractéristiques du concept (Cohen, Manion \& Morrisson, 2000). Or, pour mesurer le degré auquel les indicateurs retenus dans la littérature représentent le concept de socialisation professionnelle, il semble nécessaire d'utiliser quatre principales notions :

- la fiabilité : l'indicateur est déterminé davantage par le concept que par le hasard ;

- la validité : l'indicateur mesure bel et bien ce qu'il est supposé mesurer ;

-la faisabilité : le nombre d'indicateurs ne doit pas être trop élevé et ceux-ci doivent être compréhensibles;

-la sensibilité : l'indicateur est-il capable d'enregistrer des variations assez fines du concept.

Dans un premier temps, nous nous attarderons aux critères en usage pour déterminer le niveau de socialisation de l'acteur. Dans un deuxième temps, nous nous pencherons - trop rapidement nous en convenons déjà - sur la manière dont le temps est pris en compte dans la mesure de la socialisation professionnelle. Précisons que cette partie de notre exposé doit énormément au travail synthèse effectué par Catherine Fabre du Laboratoire Interdisciplinaire de recherche sur les Ressources Humaines et l'Emploi (LIRHE, Unité mixte de recherche CNRS/UT1 Université des Sciences Sociales, Toulouse). Nous empruntons donc sensiblement les mêmes chemins que ceux de cette chercheure. 


\section{Les indicateurs de la socialisation professionnelle}

Tout d'abord, nous allons présenter les indicateurs qui représentent les conséquences attitudinales de la socialisation. Ensuite, nous nous attarderons aux indicateurs qui traduisent les conséquences de la socialisation en termes de maîtrise des domaines de socialisation. Enfin, nous analyserons les indicateurs donnant à voir l'aspect dynamique de la socialisation professionnelle.

\subsection{Typologie et caractéristiques des mesures effectuées}

La très grande majorité des mesures de la socialisation professionnelle porte sur les conséquences de la socialisation. En fait, on analyse directement l'effet de cet indicateur sur d'autres variables telles la satisfaction au travail, l'engagement professionnel, le projet de quitter son emploi, le rôle professionnel, etc. Ces dernières variables sont censées représenter fidèlement le niveau de socialisation professionnelle du travailleur.

À l'instar de Fabre (2005), on peut alors se poser deux questions : 1- Dans quelle mesure les conséquences sont-elles réellement corrélées à la socialisation professionnelle ? 2- Est-il suffisant de mesurer les conséquences de la socialisation professionnelle? Expliquons brièvement. La première question renvoie au problème suivant : considérer implicitement que l'insertion professionnelle dépend uniquement de la socialisation professionnelle. Or, des recherches en ce domaine démontrent bien que ce n'est pas le cas (Martineau, Vallerand, \& Bergevin, 2008; Portelance, Mukamurera, Martineau \& Gervais, 2008; Vallerand \& Martineau, 2006). Bien des indicateurs interviennent : formation antérieure, type de contrat d'embauche, type de poste occupé, etc. On serait en droit de s'attendre à ce qu'un instrument de mesure de la socialisation professionnelle puisse indiquer clairement ce qui relève du processus de socialisation et ce qui relève d'autres facteurs. La deuxième question, quant à elle, renvoie au fait que le processus de socialisation professionnelle est à toute fin pratique considéré comme une boîte noire. En effet, les recherches ne mesurent pas ce qui se passe à l'intérieur du processus mais seulement ses résultats : satisfaction au travail, engagement professionnel, maîtrise des savoirs et des compétences, etc. Comme le souligne pertinemment Fabre $(2005,7)$ à la suite de Chao, O'Leary-Kelly, Wolf, Klein et Gardner (1994) : " En effet, ces indicateurs sont en mesure d'établir des liens de corrélation entre un facteur et un degré de réussite de la socialisation professionnelle, mais ils sont incapables d'expliquer les causes de succès ou d'échec et d'identifier les problèmes à résoudre. Constater des corrélations sans les expliquer ne permet pas d'analyser une situation, d'établir un diagnostic et des prescriptions ".

\subsection{La socialisation professionnelle mesurée par des variables de résultat}

Dans la grande majorité des recherches menées à ce jour, les variables expliquées par la socialisation professionnelle sont en fait utilisées comme des indicateurs (par exemple, la satisfaction au travail, l'intention de demeure en poste, l'engagement), des variables manifestes de ce concept. La mobilisation de ces indicateurs relativement éloignés du concept de socialisation professionnelle permet, il faut bien le dire, la multiplication des mesures empiriques. C'est la raison de leur emploi fréquent. Le problème vient du fait que peu à peu la mesure se substitue au concept lui-même (Allen \& Meyer, 1990, Ashford \& Saks, 1996 ; Jones, 1986). Force est de constater que les variables de type "attitudes " 
Le développement professionnel : quels indicateurs?

(comme celles que nous venons de mentionner) ne sont reliées que de manière fort imparfaite à la socialisation professionnelle. Par ailleurs, pour chacune il faut également identifier des indicateurs précis. En combinant ces variables pour atteindre la socialisation professionnelle et en identifiant leurs indicateurs, on multiplie les approximations dans les mesures. On peut donc se demander si l'emploi de telles variables est une pratique vraiment pertinente. En somme, compte tenu de ce qui précède, on peut se demander si ce qui est mesuré ainsi est réellement en adéquation avec le concept. En l'absence de la certitude que les variations des variables expliquées (intention de changer d'emploi, satisfaction au travail, engagement professionnel, etc.) sont effectivement expliquées par la variation de la variable indépendante (ici, la socialisation professionnelle), il est impossible d'affirmer que ce qui devrait être mesuré l'est bel et bien. En termes de validité donc, on ne peut que constater la faiblesse des outils de mesure.

\subsection{La socialisation professionnelle mesurée au moyen de domaines de socialisation}

Afin d'éliminer ou de réduire les lacunes identifiées plus haut, Chao et al. (1994) ont élaboré une mesure spécifique du construit théorique de la socialisation professionnelle. En effet, leur échelle se compose de six dimensions partiellement indépendantes : la maîtrise des compétences, le développement de relations sociales, l'acceptation de la culture organisationnelle, la maîtrise du langage de la profession et du jargon organisationnel, la capacité à utiliser les structures de pouvoir formelles et informelles, la connaissance historique de l'organisation. À l'inverse de Fisher (1986), les travaux de Chao et al. (1994) laissent cependant de côté la construction d'une identité professionnelle (ce que nous déplorons). L'échelle ainsi créée (comprenant en tout 34 items) a été testée et améliorée et présenterait un bon niveau de fiabilité et de validité interne. Selon ce qu'en rapporte notamment Fabre (2005), la variance expliquée par les six dimensions extraites lors de l'analyse en composantes principales serait de $58 \%$, et restituerait les dimensions construites théoriquement. II semblerait que cette échelle de mesure soit capable de saisir plus finement le phénomène de la socialisation professionnelle que les variables de résultat. On peut toutefois souhaiter que cette échelle soit enrichie par une variable comme l'identité professionnelle qui est, selon bien des chercheurs, associée à la socialisation professionnelle (Bauer, Morrison \& Callister, 1998). Par ailleurs, les instruments de mesure de la socialisation devraient notamment permettre de discriminer différents phénomènes. On pense ici, entre autres, au fait de comprendre son milieu de travail (par exemple, savoir qui fait quoi) et au fait d'adhérer aux valeurs du milieu (par exemple, partager le projet de son école). Signalons que d'autres chercheurs ont également élaboré des échelles du même type. On pense notamment aux travaux de Taormina (1994, 1997, 2004). Compte tenu de l'espace qui nous est imparti, il ne saurait être question ici de les présenter.

En somme, si certains travaux apportent plus de précisions dans la mesure des indicateurs de socialisation professionnelle, nous sommes encore loin de posséder des outils parfaits. On notera surtout que les items qui mesurent le degré de compréhension, ceux qui mesurent le degré d'adhésion et, enfin, ceux qui mesurent le processus comme tel, semblent insuffisamment discriminés. 
Stéphane Martineau, Liliane Portelance, Annie Presseau

La socialisation au travail : analyse des approches basées sur la mesure

\subsection{La socialisation professionnelle mesurée par son processus}

Les études que nous venons d'évoquer se centrent essentiellement sur les conséquences attendues de la socialisation professionnelle. À aucun moment le processus même de la socialisation professionnelle est réellement décrit. Toutefois, quelques chercheurs se sont penchés sur la question (Feldman, 1976 ; Louis, 1980 ; Schein, 1978). Bien que leurs travaux datent déjà de presque trois décennies, leur pertinence apparaît encore évidente (à tout le moins dans l'univers de la recherche de la mesure). Ces chercheurs ont tenté de décrire le plus précisément possible les étapes que traverse un acteur lors de sa socialisation au travail. II y aurait ainsi trois grandes étapes de socialisation : la socialisation anticipée, la confrontation à la réalité, l'adaptation.

Ces travaux nous paraissent intéressants dans la mesure où ils se centrent sur ce que vit l'acteur et ont recours à différents concepts pour le faire: les attentes envers le milieu professionnel, une vision réaliste du milieu de travail, les conflits d'identité, le changement, l'adaptation à de nouvelles valeurs, la perception et l'interprétation de l'information. Par contre, ces travaux n'expliquent pas les mécanismes de passage entre les différentes étapes, ce qui est une lacune importante sur le plan de la compréhension du processus de socialisation professionnelle. Par ailleurs, les écrits de ces chercheurs demeurent fort peu explicites quant à la durée des étapes de socialisation (et donc sur les raisons expliquant cette durée).

\section{Le temps dans la mesure de la socialisation professionnelle}

Bien qu'il semble aller de soi que le temps joue un rôle significatif dans le processus de socialisation professionnelle, cet indicateur a été étonnamment négligé par les recherches. En effet, même si de nombreuses études longitudinales ont été menées depuis deux décennies sur la socialisation professionnelle (Bauer et al. 1998), elles ont généralement laissé le facteur temps dans une sorte d'arrière plan théorique (Shuval \& Adler, 1977). II semble pourtant nécessaire d'inclure l'effet du temps dans la mesure de la socialisation au travail (Fabre, 2005). Les recherches longitudinales soulèvent ainsi un certain nombre de questions : Doivent-elles se limiter à la seule première année d'embauche (Hill 1992) ? Et, dans ce cas, comment peuvent-elles prendre en compte le type de lien d'emploi (par exemple, les enseignants à temps partiel, ceux à contrat d'une durée limitée, les enseignants à la leçon, etc.). Sachant que la socialisation professionnelle est un processus irrégulier (Fabre, 2005; Pinder \& Schroder, 1987), comment les dispositifs de recherche longitudinale peuvent-ils, sur le plan méthodologique, prendre en compte cette irrégularité ?

Force est de constater alors que notre compréhension du processus de socialisation professionnelle est encore à parfaire. Ainsi, si on sait que les attitudes et les perceptions prennent forme assez tôt dès l'entrée au travail et même avant (Portelance, Mukamurera, Martineau \& Gervais, 2008), on en sait encore trop peu sur la façon réelle dont ces dernières se construisent et selon quelle temporalité. Comme nous le rappelle Fabre $(2005,15)$ : « II semble également nécessaire d'étudier le processus de socialisation en profondeur, pour trouver un modèle explicatif de la dynamique temporelle de la socialisation et comprendre les déterminants du progrès, les évènements qui peuvent accélérer ou au contraire freiner la progression ". Les recherches menées à ce jour ont trop tendance à présenter le processus de socialisation professionnelle d'une manière linéaire (Langley, 1999). Pourtant, la construction théorique de Dubar (2000), laisse bien voir que le processus de socialisation ne 
Le développement professionnel : quels indicateurs?

saurait être conçu en tant que processus continu et linéaire. Au contraire, la socialisation professionnelle semble aussi faite de moments de discontinuité, de tensions, de contradictions voire même de ruptures (Mintzberg, Raisinghani \& Théorêt, 1976; Perier, 2004 ; Schwenk, 1985 \& Tremblay, 1998). Qu'on nous permette une fois de plus de citer un peu longuement le texte de Fabre $(2005,16)$ : "Actuellement, les données longitudinales sont prélevées alors que l'on ne maîtrise pas la dynamique du processus. Ainsi, dans l'hypothèse où les différents domaines de socialisation ne progressent pas au même rythme, et où l'on prélèverait des données à un moment où l'individu ressent un retour en arrière dans l'un des domaines, les résultats ne signifieraient absolument rien. Par conséquent, il serait intéressant d'intégrer, au sein d'un modèle unique, théories de la variance et théories du processus ".

Des recherches qualitatives semblent nécessaires ici afin de raffiner notre compréhension du processus de socialisation professionnelle. Ces recherches pourraient fournir des pistes intéressantes pour la construction d'indicateurs plus fins tant en ce qui concerne les résultats du processus qu'en ce qui a trait au processus lui-même. Par ailleurs, les recherches qualitatives pourraient également aider les chercheurs à élaborer des approches méthodologiques mieux ajustées à la complexité du processus analysé. On ne peut que déplorer qu'à ce jour (comme c'est souvent le cas dans nombre de domaines en sciences humaines et sociales), les recherches dites qualitatives (à tendance phénoménologique, interactionniste ou ethnométhodologique, pour ne nommer que ces approches) et les recherches disons plus quantitatives (faisant usage d'échelles standardisées) aient été menées dans une relative ignorance mutuelle.

\section{Mais, en fin de compte, est-il pertinent de mesurer la socialisation professionnelle ?}

On le sait, "l'épistémologie de la mesure " ${ }^{2}$ (ou, si l'on préfère, le courant hypothéticodéductif) possède une longue tradition et, malgré bien des faiblesses, a développé des outils méthodologiques souvent performants qui ne sont pas sans intérêt (comme on a pu le voir plus haut). Or, nos propos précédents ont porté sur les aspects essentiellement méthodologiques des outils de mesure de la socialisation professionnelle laissant volontairement de côté la question des fondements épistémologiques. C'est de cet aspect du problème dont il sera question dans cette section. On nous excusera de la brièveté de notre réflexion, l'espace qui nous est imparti étant relativement restreint.

Les tensions entre les approches positivistes et les approches interprétatives traversent toute l'histoire des sciences humaines et sociales et ce, par exemple, dès les productions pionnières en sociologie de Durkheim et de Weber (Delas \& Milly, 2005 ; Simon, 1991). En la matière, Vérité et méthode de Gadamer (1996, paru originellement en 1960) a bien montré non seulement les limites mais aussi les risques d'une «importation " des approches des sciences naturelles dans les sciences humaines et sociales. Plus encore, le grand philosophe allemand nous a mis en garde contre une conception substantialiste des concepts car le langage ne donne pas à voir un monde ontologique préexistant mais fait plutôt apparaître dans l'unité du "vouloir dire " le monde qu'il constitue. En fait, plus spécifiquement, pour comprendre les phénomènes humains, il faut comprendre le sens que leur attribuent les sujets concernés. C'est dire qu'il faut tenir compte des fins poursuivies par les sujets (Schutz,

\footnotetext{
2 Nous reprenons ici l'expression du professeur Pierre Paillé de l'Université de Sherbrooke.
} 
Stéphane Martineau, Liliane Portelance, Annie Presseau

La socialisation au travail : analyse des approches basées sur la mesure

1987). Le sujet est alors considéré comme une unité psychique, un ensemble compréhensible, qui possède une structure, une certaine permanence dans le temps et en qui on peut voir à l'œuvre des processus intelligibles (Watier, 2002). Ajoutons que l'approche interprétative accorde une grande importance à certains facteurs généralement ignorés par les conceptions hypothético-déductive au chapitre desquels on note: 1- un sujet qui interprète et qui est situé socialement, culturellement, historiquement ; 2- une pratique sociale de l'interprétation qui est historiquement ancrée ; 3- une action nécessairement située ; 4non seulement l'interprété mais aussi l'interprétant sont marqués par la temporalité. Qu'en est-t-il dans les approches présentées plus haut? Au regard d'une épistémologie interprétative, on l'aura deviné, les approches de la mesure de la socialisation professionnelle posent plusieurs problèmes.

Elles se sont globalement développées en dehors de toute réflexion sérieuse sur la place et le rôle du chercheur. Les recherches de la mesure de la socialisation professionnelle n'ont pas donné lieu à un questionnement sur leur ancrage historique (par exemple, en quoi participent-elles d'un courant néo-libéral de gestion de la main-d'œuvre et de la gouvernance des organisations ?). Elles semblent travailler à partir d'une définition a priori du concept, définition qui fige le concept qu'elles investiguent. Elles découpent en outre le processus en différents facteurs qu'elles analysent plus ou moins séparément sans prendre en compte le cadre herméneutique où évoluent les sujets; nous sommes loin ici d'une approche holistique. De plus, comme nous l'avons indiqué dans une section précédente, elles ne tiennent pas (ou peu) compte du facteur temps. En effet, la socialisation est un processus qui se déroule dans un laps de temps relativement long que les approches de la mesure peuvent difficilement prendre en compte. Par ailleurs, elles font l'impasse sur les dimensions conflictuelles, les tensions, les rapports stratégiques entre les acteurs donnant plutôt à voir un phénomène essentiellement linéaire.

En fin de compte, quelle réponse donner à l'intitulé de cette section? Ici, nous sommes tentés d'adopter une position similaire à celle de Ricœur $(1969 ; 1983,1986)$ face à la controverse entre les tenants de l'explication et ceux de la compréhension en sciences humaines et sociales à savoir que devant les phénomènes humains, l'explication de processus (sur un mode hypothético-déductif) peut contribuer à améliorer la compréhension que nous en avons au sens où, par exemple, la connaissance que j'ai de certains processus cognitifs du cerveau peut m'aider à mieux comprendre la situation d'apprentissage de tel ou tel élève. Si les phénomènes humains, pour être intelligibles, nécessitent la prise en compte du sens construit par les acteurs (posture compréhensive), ils n'impliquent pas un rejet complet et systématique de toute visée explicative. Chacune des approches comporte des limites. En somme, les recherches sur la mesure ne sont pas sans intérêt pour l'explication de la socialisation professionnelle, elles permettent de mettre au jour différentes dimensions en jeu dans ce processus. Toutefois, au-delà de leurs lacunes méthodologiques (leur relative incapacité à mesurer réellement le processus), leur posture épistémologique leur interdit de prendre en compte les raisons d'agir, les motifs, les logiques des acteurs et, en cela, leur portée explicative s'en trouve limitée.

\section{Conclusion}

Ce tour d'horizon est bien entendu trop bref et ne saurait rendre toutes les subtilités du champ de recherche présenté. Mais, malgré ces limites indéniables, ce texte permet, nous le 
Le développement professionnel : quels indicateurs?

croyons, de faire ressortir certaines caractéristiques de la recherche sur la socialisation professionnelle conduite à partir d'outils de mesure.

II ressort donc de notre présentation que les indicateurs mobilisés pour étudier la socialisation sont passablement imparfaits. En fait, certains ne sont même pas liés au concept, tandis que d'autres manquent singulièrement de rigueur. On peut donc s'interroger sur la portée des résultats obtenus à partir de tels outils imparfaits. II apparaît alors nécessaire de développer des instruments appropriés pour comprendre non seulement la réussite ou l'échec de la socialisation, mais également pour juger, par exemple, de l'efficacité des programmes et des dispositifs d'insertion professionnelle en milieu scolaire (Martineau \& Portelance, 2005). Pour ce faire, une étude approfondie du processus même de socialisation professionnelle dans un esprit interprétatif et par le biais de méthodologies qualitatives nous semble une avenue nécessaire. En somme, au-delà des faiblesses méthodologiques, c'est l'esprit même dans lequel sont menées les recherches de la mesure qui est questionnable. En effet, celles-ci adoptent une posture plutôt positiviste qui nous semble réductrice et peu compatible avec la complexité du phénomène.

\section{Bibliographie}

Adler, P.A. \& Adler, P. (2005). The identity career of the graduate student: Professional socialization to academic sociology. New Brunswick, NJ: Transaction Periodicals Consortium. Allen, N.J. \& Meyer, J.P. (1990). Organizational socialization tactics: A longitudinal analysis of links to newcomers' commitment and role orientation. Academy of Management Journal, 33, 847-858.

Ashford, S.J. \& Saks A.M. (1996). Socialization tactics: Longitudinal effects on newcomers adjustment. Academy of Management Journal, 39(1), 149-178.

Bauer, T.N., Morrison, E.W. \& Callister, R.R. (1998). Organizational Organization: A Review and Directions for Future Research. Research in Personnel and Human Resource Management, 16, 149-214.

Bolliet, D. \& Schmitt, J.-P. (2002). La socialisation. Paris : Bréal.

Bryant, C.C. (2007). A study of the evaluation procedures for professional development among public school districts in Virginia, Thèse de doctorat. Virginia: The college of William and Mary in Virginia.

Chao, G.T., O'Leary-Kelly, A.M., Wolf, S., Klein, H.K., \& Gardner, P.D. (1994). Organizational Socialization: Its Content and Consequences. Journal of Applied Psychology, 79, 730-743.

Clark, P.G. (1997). Values in health care professional socialization: Implications for geriatric education in interdisciplinary teamwork. Gerontologist, 4, 441-451.

Cohen, L., Manion, L. \& Morrisson, K. (2000). Research Methods in Edu-cation. London / New York: Routledge.

Day, C. (1999). Developing teachers: the challenges of lifelong learning. London: Falmer.

Delas, J.-P. \& Milly, B. (2005). Histoire des pensées sociologiques. Paris : Armand Colin.

D'lribarne, P. (1986). Vers une gestion culturelle des entreprises, Gérer et Comprendre. Annales des mines, 4, 72-85.

Dixon, C. (2005). African American women in higher education administration: their professional position and relationship of professional socialization and systematic barriers in their carrier path. Thèse de doctorat. Minneapolis: Capella University. 
Dubar, C. (1996). La socialisation : construction des identités sociales et professionnelles. Paris : Armand Colin.

Dubar, C. \& Tripier, P. (2005). Sociologie des professions. Paris : Armand Colin.

Dumont, F. (1968). Le lieu de l'homme. La culture comme distance et mémoire. Montréal: Hurtubies $\mathrm{HMH}$.

Dunn, D., Linda, R. \& Seff, A. (1994). New Faculty Socialization in the academic workplace, In C. Smart (Ed.) Higher Education: Theory and Research, 10. New York: Agathon, 374-416. Durkheim, E. (1967). De la division du travail social. Paris : PUF.

Fabre, C. (2005). Décentralisation à la Française. Critique et contribution à la mesure de la socialisation organisationnelle en recherche de gestion. Toulouse: Les notes du LIRHE [http://www.univ-tlse1.fr/LIRHE/]

Feldman, D.C. (1976). A Contingency Theory of Socialization. Administrative Science Quarterly, 21, 433-452.

Fisher, C.D. (1986). Organizational socialization: an integrative review. Research in personnel and Human Resource Management, 4, 101-145.

Freitag, M. (2002). L'oubli de la société. Pour une théorie critique de la postmodernité. Québec : Les Presses de l'Université Laval.

Gadamer, H.-G. (1996). Vérité et méthode. Paris : Seuil.

Giddens, A. (2005). La construction de la société. Paris: PUF.

Guillemette, F. (2006). L'engagement des enseignants du primaire et du secondaire dans leur développement professionnel, Thèse de doctorat. Trois-Rivières : Université du Québec à Trois-Rivières.

Gundry, L.K. (1993). Fitting into technocal organizations: The socialization of newcomers engineers. IEEE Transactions on engineering management, 40, 335-345.

Guskey, T.R. (2000). Evaluating professional development. Thousand Oaks, CA: Corwin Press.

Hébrard, P. (2004). Discussion et socialisation professionnelle. In M. Tozzi, \& R. Etienne (Ed.), La discussion en éducation et en formation: un nouveau champ de recherches. Paris : L'Harmattan, 167-192.

Helm, M.P. (2004). Professional identity, sense-making, and the market effect: perspectives from new student affairs professionals, Thèse de doctorat. Arizona: The University of Arizona. Hill, L.A. (1992). Becoming a manager. Boston: Harvard Business School Press.

Høivik $H$. (2005). Aspects critiques de la socialisation professionnelle des étudiants de première année en école de bibliothéconomie. Oslo University College. [http://www.ifla.org/lV/ifla71/papers/058f_trans-Hoivik.pdf]

Jones, G. (1986). Socialization Tactics, Self-Efficacy, and Newcomers' Adjustments to Organizations. Academy of Management Journal, 29, 262-279.

Keith, B., Moore, H.A. (1995). Training sociologists: an assessment of professional socialization and the emergence of career aspirations. Washington, DC: American Sociological Association.

Killion, J. (2002). Assessing Impact: Evaluating staff development. Oxford, OH: National Staff Development Council.

Klossner, J.T. (2004). "Becoming" an athletic trainer: the professional socialization of preservice athletic trainers. Thèse de doctorat. Indiana: Indiana University. 
Le développement professionnel : quels indicateurs?

Lacaze, D. (2002). Le rôle de l'individu dans la socialisation organisationnelle, Thèse de doctorat. Marseille : Université Aix-Marseille III.

Lacey, C. (1994). Teachers, Professional socialization of. In T. Husen \& T. Rostlethwaite (Ed.), The International Encyclopedia of Education. Exeter: Pergamon, 6122-6126.

Langley, A., (1999). Strategies for theorizing from process data. Academy of Management Review, 24(4), 691-710.

Langlois, J. (2000). Une étude de la socialisation professionnelle par la distinction de l'identité professionnelle des artistes formes et autodidactes, Thèse de doctorat. Montréal : Université de Montréal.

Linton, R. (1986). Le fondement culturel de la personnalité. Paris : Dunod. (1 édition 1945).

Louis, M.R. (1980). Surprise and Sense Making: What Newcomers Experience in Entering Unfamiliar Organizational Settings. Administrative Science Quarterly, 25, 226-251.

Martineau, S. (2008). L'identité professionnelle et l'insertion professionnelle: essai de problématique. Formation et profession, 15(1), 40-43.

Martineau, S. \& Portelance, L. (2005). L'insertion professionnelle: un tour d'horizon des recherches. L'Écho du RÉS.É.A.U., 5(1), 7-17.

Martineau, S. \& Presseau, A. (2007). Se "mettre en mots " ou se bricoler une histoire pour qu'elle prenne sens : le discours identitaire d'enseignants du secondaire. In C. Gohier (Ed.) Identités professionnelles d'acteurs de l'enseignement. Regards croisés, Presses de I'Université du Québec., 67-88.

Martineau, S., Presseau, A. \& Portelance, L. (2009). Analyse d'un programme d'insertion professionnelle dans une commission scolaire québécoise. Rapport de recherche présenté au Conseil de recherche en sciences humaines du Canada (CRSH). Carrefour national de l'insertion professionnelle en enseignement [http://www.insertion.qc.ca/spip.php?article213] [http://www.insertion.qc.ca/IMG/pdf_Rapport_de_recherche_LADIPE.pdf]

Martineau, S. \& Vallerand, A.-C. (2007). Les dispositifs pour soutenir l'insertion professionnelle des enseignants. Carrefour national de l'insertion professionnelle en enseignement (CNIPE).[http://www.insertion.qc.ca/arti-cle.php3?id_article=134] [http://www. insertion.qc.ca//MG/pdf/Martineau_dispositifs.pdf]

Martineau, S., Vallerand, A.-C \& Bergevin, C. (2008). Portrait thématique des écrits sur l'insertion professionnelle en enseignement. In Portelance, Mukamurera, Martineau \& Gervais (Ed.), L'insertion dans le milieu scolaire : une phase cruciale du développement professionnel de l'enseignant, Québec : Les Presses de l'Université Laval, 11-30.

Mead, G.H. (1963). L'esprit, le soi, la société. Paris : PUF.

Mintzberg, H., Raisinghani, D. \& Théorêt, A. (1976). The structure of unstructured decision processes. Administrative Science Quarterly, 21, 246-275.

Nault, G. (2005). Étude du fonctionnement et du potentiel d'une communauté de pratique en ligne pour le développement professionnel d'enseignants novices. Thèse de doctorat. Montréal : Université du Québec à Montréal.

Osiek-Parisod, F. (1995). Les infirmières scolaires: malaise identitaire et socialisation professionnelle. Zürich: Seismo.

Piaget, J (1965). L'explication en Sociologie, Études Sociologiques. Paris : Droz.

Pinder, C.C. \& Schroeder, K.G. (1987). Time to proficiency following job transfers. Academy of Management Journal, 30, 336-353. 
Portelance, L., Mukamurera, J., Martineau, S. \& Gervais, C. (Ed.) (2008). L'insertion dans le milieu scolaire: une phase cruciale du développement professionnel de l'enseignant. Québec : PUL.

Ricœur, P. (1969). Le conflit des interprétations. Essais d'herméneutique. Paris : Seuil.

Ricœur, P. (1983). Temps et récit. L'intrigue et le récit historique. Paris : Seuil.

Ricœur, P. (1986). Du texte à l'action. Essais d'herméneutique II. Paris : Seuil.

Rocher, G. (1992). Introduction à la sociologie. Montréal : Hurtubise HMH. (1ère édition 1969).

Sainsaulieu, R. (1977). L'identité au travail. Presses de la Fondation Nationale des Sciences Politiques.

Sainsaulieu, R. (1984). Renouveau des cultures d'entreprise ? Projet, 183, 294-304.

Schein, E.H. (1978). Career Dynamics: Matching Individual and Organizational Needs, Addison-Wesley Publishing Co., 112-127.

Schutz, A. (1987). Le chercheur et le quotidien. Paris: Klincksieck.

Schwenk, C. (1985). The use of participant recollection in the modelling of organizational processes. Academy of Management Review, 10, 496-503.

Shamatov, D. A. (2005). Beginning teachers' professional socialization in post-soviet Kyrgyzstan: challengers and coping strategies, Thèse de doctorat. Toronto: University of Toronto.

Shuval, J.T. \& Adler, I. (1977). Processes of continuity and change during socialization for medecine in Israël. Journal of Health and Social Behavior, 18, 112-124.

Simon, P.-J. (1991). Histoire de la sociologie. Paris : PUF.

Speck, M. \& Knipe, C. (2001). Why can't we get it right: Professional development in our schools. Thousand Oaks, CA: Corwin Press.

Taormina, R.J. (1994). The Organizational Socialization Inventory. International Journal of Selection and Assessment, 2(3),133-145.

Taormina, R.J. (1997). Organizational Socialization: a multi-domain, continuous process model. International Journal of Selection and Assessment, 5(1), 29-47.

Taormina, R.J. (2004). Convergent validation of two measures of organizational socialization. International Journal of Human Resource Management, 15 (1), 76-94.

Tremblay, N. (1998). Socialisation et cheminement professionnel d'enseignantes et de directrices du primaire. Histoires de vie et trajectoires professionnelles, Thèse de doctorat. Chicoutimi (Canada) : Université du Québec à Chicoutimi.

Uwamariya, A. \& Mukamurera, J. (2005). " Le concept de développement professionnel » en enseignement: concept théorique " Revue des Sciences de l'éducation, v.31, no 1, 2005, 133-155.

Vallerand, A.-C.\& Martineau, S. (2006). Recension des écrits sur l'insertion professionnelle en enseignement. 575 pages. Carrefour national de l'insertion professionnelle en enseignement (CNIPE). [http://www.insertion.qc.ca/article.php3?id_article=58]

Van Maanen, J. \& Schein, E.H. (1979). Toward a theory of organizational socialization. Research in Organizational Behavior, 1, 209-264.

Watier, P. (2002). Une introduction à la sociologie compréhensive. Belval : Circé.

Weber, M. (1971). Économie et Société. Paris: Plon. (Traduction partielle de l'original allemand paru en 1921) 